\title{
Against Phase Velocities of Elastic Waves in Thin Orthotropic Cylindrical Shell
}

\author{
A.A. Kleshchev \\ St. Petersburg State Marine Technical University, st. Lotsmanskaya 3, St. Petersburg, 190008, Russia \\ *Corresponding Author: alexalex-2@yandex.ru
}

Copyright (C) 2013 Horizon Research Publishing. All rights reserved.

\begin{abstract}
In paper is received the characteristic equation for the determine of wave numbers of phase velocities of elastic waves in the thin cylindrical shell with the help of the dynamic theory of the elasticity for the orthotropic medium and of the hypothesis of thin shells.
\end{abstract}

Keywords Theory of Elasticity, Phase Velocity, Wave Number, Characteristic Equation, Boundary Conditions

\section{Introduction}

Based at the use of the dynamic theory of the elasticity for the anisotropic medium and with the help of the hypothesis of thin shells is determined the characteristic equation for wave numbers of elastic waves in the thin orthotropic cylindrical shell.

\section{The Dynamic Theory of the Elasticity for the Orthotropic Medium}

Let's consider the infinite thin orthotropic cylindrical shell. The harmonic elastic wave is spread along axis $Z$, that is the axis of the symmetry of the second order. The orthotropic elastic medium is characterized by the nine elastic moduluses $[1-4]$ :

$$
A_{11}, A_{12}, A_{13}, A_{22}, A_{23}, A_{33}, A_{44}, A_{55}, A_{66} \text {. }
$$

Components of the displacement vector $\vec{U}\left(U_{r}, U_{\varphi}, U_{z}\right)$ can be presented in the form of the serieses [2-4]:

$$
\begin{gathered}
U_{r}=\exp (i \bullet k \cdot z) \cdot \sum_{m=0}^{\infty} \cos (m \bullet \varphi) \cdot U_{m}(r) ; \\
U_{\varphi}=\exp (i \bullet k \cdot z) \cdot \sum_{m=1}^{\infty} \sin (m \bullet \varphi) \bullet V_{m}(r) ;
\end{gathered}
$$

$$
U_{z}=\exp (i \cdot k \cdot z) \cdot \sum_{m=0}^{\infty} \cos (m \cdot \varphi) \cdot W_{m}(r),
$$

where $k$ is the wave number of the elastic wave [2 - 4].

Equations of the dynamic balance in displacements are [2 4]:

$$
\begin{gathered}
\frac{\partial^{2} U_{r}}{\partial r^{2}}+\frac{a_{1}}{r} \cdot \frac{\partial^{2} U_{\varphi}}{\partial \varphi \partial r}+a_{2} \cdot \frac{\partial^{2} U_{z}}{\partial z \partial r}+ \\
\frac{a_{3}}{r} \cdot \frac{\partial^{2} U_{\varphi}}{\partial \varphi \partial r}-\frac{a_{3}}{r} \cdot \frac{\partial U_{\varphi}}{\partial \varphi}+\frac{a_{3}}{r^{2}} \cdot \frac{\partial^{2} U_{r}}{\partial^{2} \varphi}+ \\
a_{4} \cdot \frac{\partial^{2} U_{r}}{\partial z^{2}}+a_{4} \cdot \frac{\partial^{2} U_{z}}{\partial r \partial z}+\frac{1}{r} \cdot \frac{\partial U_{r}}{\partial r}+ \\
+\frac{a_{2}}{r} \cdot \frac{\partial U_{z}}{\partial r}-\frac{a_{5}}{r^{2}} \cdot U_{r}-\frac{a_{6}}{r} \cdot \frac{\partial U_{z}}{\partial z}+a_{7} \cdot U_{r}=0
\end{gathered}
$$

where

$$
a_{1}=\frac{A_{12}}{A_{11}} ; a_{2}=\frac{A_{13}}{A_{11}} ; a_{3}=\frac{A_{66}}{A_{11}} ; a_{4}=\frac{A_{55}}{A_{11}} ; a_{5}=\frac{A_{22}}{A_{11}} ;
$$

$a_{6}=\frac{A_{23}}{A_{11}} ; a_{7}=\frac{\rho \omega^{2}}{A_{11}} ; \rho-$ the density of the ma-terial of the shell; $\omega-$ the angular frequency.

$$
\begin{gathered}
\frac{\partial^{2} U_{\varphi}}{\partial r^{2}}+\frac{1}{r} \cdot \frac{\partial^{2} U_{r}}{\partial \varphi \partial r}+\frac{a_{8}}{r} \cdot \frac{\partial^{2} U_{r}}{\partial r \partial \varphi}+\frac{a_{9}}{r} \cdot \frac{\partial^{2} U_{\varphi}}{\partial \varphi^{2}}+ \\
\frac{a_{9}}{r} \cdot \frac{\partial U_{r}}{\partial \varphi}+\frac{a_{10}}{r} \cdot \frac{\partial^{2} U_{z}}{\partial z \partial \varphi}+\frac{a_{11}}{r} \cdot \frac{\partial^{2} U_{z}}{\partial \varphi \partial z}+ \\
a_{11} \cdot \frac{\partial^{2} U_{\varphi}}{\partial z^{2}}+\frac{1}{r} \cdot \frac{\partial U_{\varphi}}{\partial r}- \\
-\frac{1}{r^{2}} \cdot U_{\varphi}+\frac{1}{r^{2}} \cdot \frac{\partial U_{r}}{\partial \varphi}+a_{12} \cdot U_{\varphi}=0,
\end{gathered}
$$

Where 


$$
\begin{aligned}
a_{8}= & \frac{A_{12}}{A_{66}} ; a_{9}=\frac{A_{22}}{A_{66}} ; a_{10}=\frac{A_{23}}{A_{66}} ; a_{11}=\frac{A_{44}}{A_{66}} ; a_{12}=\frac{\rho \omega^{2}}{A_{66}} . \\
& \frac{\partial^{2} U_{r}}{\partial z \partial r}+\frac{\partial^{2} U_{z}}{\partial r^{2}}+\frac{a_{13}}{r^{2}} \cdot \frac{\partial^{2} U_{z}}{\partial \varphi^{2}}+\frac{a_{13}}{r} \cdot \frac{\partial^{2} U_{\varphi}}{\partial z \partial \varphi}+ \\
& a_{14} \cdot \frac{\partial^{2} U_{r}}{\partial r \partial z}+\frac{a_{15}}{r} \cdot \frac{\partial^{2} U_{\varphi}}{\partial \varphi \partial z}+\frac{\left(a_{15}+1\right)}{r} \cdot \frac{\partial U_{r}}{\partial z}+\text { (4) } \\
& \frac{1}{r} \cdot \frac{\partial U_{z}}{\partial r}+a_{16} \cdot \frac{\partial^{2} U_{z}}{\partial z^{2}}+a_{17} \cdot U_{z}=0
\end{aligned}
$$

where

$$
a_{13}=\frac{A_{44}}{A_{55}} ; a_{14}=\frac{A_{13}}{A_{55}} ; a_{15}=\frac{A_{23}}{A_{55}} ; a_{16}=\frac{A_{33}}{A_{55}} ; a_{17}=\frac{\rho \omega^{2}}{A_{55}} \text {. }
$$

Now if components of the displacement vector $\vec{U}$ taken from (1) substitute in (2) - (4), then we receive fol-lowing equations for radial functions $U_{m}(r), V_{m}(r), W_{m}(r)$ [2]:

$$
\begin{aligned}
& \frac{\partial^{2} U_{m}}{\partial r^{2}}+\frac{m \cdot\left(a_{1}+a_{3}\right)}{r} \cdot \frac{\partial V_{m}}{\partial r}+\left(a_{2}+a_{4}\right) \cdot i \cdot k \cdot \frac{\partial W_{m}}{\partial r}- \\
& \frac{m \cdot\left(a_{3}+a_{5}\right)}{r^{2}} \cdot V_{m}-\frac{a_{3}}{r^{2}} \cdot m^{2} \cdot V_{m}-a_{4} \cdot k^{2} \cdot U_{m}+\frac{1}{r} \cdot \frac{\partial U_{m}}{\partial r}+ \\
& +\frac{\left(a_{2}-a_{6}\right)}{r} \bullet i \cdot k \cdot W_{m}+\left(a_{7}-\frac{a_{5}}{r^{2}}\right) U_{m}=0 ; \\
& \frac{\partial^{2} V_{m}}{\partial r^{2}}-\frac{m \cdot\left(1+a_{8}\right)}{r} \cdot \frac{\partial U_{m}}{\partial r}-\frac{m^{2} \cdot a_{9}}{r} \cdot V_{m}- \\
& \frac{m \cdot a_{9}}{r} \cdot U_{m}+\frac{1}{r} \cdot \frac{\partial V_{m}}{\partial r}-\frac{m \bullet\left(a_{10}+a_{11}\right)}{r} \cdot i \cdot k \cdot W_{m}- \\
& a_{11} \cdot k^{2} \cdot V_{m}+\left(a_{12}-\frac{1}{r^{2}}\right) \cdot V_{m}-\frac{m}{r^{2}} \cdot U_{m}=0 ; \\
& \left(1+a_{14}\right) \cdot i \cdot k \cdot \frac{\partial U_{m}}{\partial r}+\frac{\partial^{2} W_{m}}{\partial r^{2}}-\frac{m^{2} \cdot a_{13}}{r^{2}} \cdot W_{m}+ \\
& m \cdot i \cdot k \cdot \frac{1}{r} \cdot\left(a_{13}+a_{15}\right) \cdot V_{m}+i \cdot k \cdot \frac{\left(1+a_{15}\right)}{r} \cdot U_{m}+ \\
& \frac{1}{r} \bullet \frac{\partial W_{m}}{\partial r}-k^{2} \bullet
\end{aligned}
$$

$$
\bullet a_{16} \cdot W_{m}+a_{17} \bullet W_{m}=0
$$

Boundary conditions [normal $\left(\sigma_{r}\right)$ and tangent $\left(\tau_{r \varphi}, \tau_{r z}\right)$ stresses are equal zero at external $(r=a)$ and in-ternal $(r=b)$ surfaces of the elastic shell] are added to equations (5) $-(7)[2]$ :

$$
\begin{gathered}
\frac{\partial U_{m}}{\partial r}+\frac{a_{1}}{r} \cdot m \bullet V_{m}+\frac{a_{1}}{r} \cdot U_{m}+a_{2} \bullet i \bullet k \cdot W_{m}=0 \\
{[\mathrm{r}=\mathrm{a} ; \mathrm{r}=\mathrm{b}]} \\
\frac{\partial V_{m}}{\partial r}-\frac{1}{r} \cdot V_{m}-\frac{m}{r} \cdot U_{m}=0 ; \quad[\mathrm{r}=\mathrm{a} ; \mathrm{r}=\mathrm{b}] \\
i \bullet k \cdot U_{m}+\frac{\partial W_{m}}{\partial r}=0 ; \quad[\mathrm{r}=\mathrm{a} ; \mathrm{r}=\mathrm{b}]
\end{gathered}
$$

\section{Hypothesis of Thin Shells}

The fellow parameter $\xi=\frac{z}{R_{0}}$ can be used for thin shells, where $R_{0}=\frac{a+b}{2}$ is the middle radius and $z=r-R_{0}$ is the coordinate taking from the middle surface [2-6]:

$$
\begin{aligned}
& U_{m}(r)=\sum_{n=0}^{N_{1}} x_{n} \bullet \xi^{n} ; \\
& V_{m}(r)=\sum_{n=0}^{N_{1}} y_{n} \bullet \xi^{n} ; \\
& W_{m}(r)=\sum_{n=0}^{N_{1}} z_{n} \bullet \xi^{n} .
\end{aligned}
$$

We substitute decompositions in boundary conditions (8) - (10) and receive 6 equations relative to $3 \cdot\left(N_{1}+1\right)$ unknown coefficients $x_{n}, y_{n}, z_{n}$ [2]: 


$$
\begin{gathered}
R_{0}^{-1} \cdot \sum_{n=0}^{N_{1}} x_{n} \bullet n \bullet\left(\xi_{1}\right)^{n-1}+a_{1} \bullet m \bullet\left(R_{0}+0,5 \cdot h\right)^{-1} \cdot \sum_{n=0}^{N_{1}} y_{n} \cdot\left(\xi_{1}\right)^{n}+a_{1} \bullet\left(R_{0}+0,5 \cdot h\right)^{-1} \sum_{n=0}^{N_{1}} x_{n} \cdot\left(\xi_{1}\right)^{n}+a_{2} \bullet i \bullet k \bullet \\
\cdot \sum_{n=0}^{N_{1}} z_{n} \cdot\left(\xi_{1}\right)^{n}=0
\end{gathered}
$$

Where

$$
\begin{aligned}
& \xi_{1}=\frac{a-R_{0}}{R_{0}} \\
& h=a-b \text {. } \\
& R_{0}^{-1} \cdot \sum_{n=9}^{N_{1}} x_{n} \bullet n \bullet\left(-\xi_{1}\right)^{n-1}+a_{1} \bullet m \cdot\left(R_{0}-0,5 \cdot h\right)^{-1} \cdot \sum_{n=0}^{N_{1}} y_{n} \bullet\left(-\xi_{1}\right)^{n}+a_{1} \cdot\left(R_{0}-0,5 \cdot h\right)^{-1} \sum_{n=0}^{N_{1}} x_{n} \bullet\left(-\xi_{1}\right)^{n}+a_{2} \bullet i \bullet k \bullet \\
& \sum_{n=0}^{N_{1}} z_{n} \cdot\left(-\xi_{1}\right)^{n}=0 \\
& R_{0}^{-1} \cdot \sum_{n=0}^{N_{1}} y_{n} \cdot n \cdot\left(\xi_{1}\right)^{n-1}-\left(R_{0}+0,5 \cdot h\right)^{-1} \cdot \sum_{n=0}^{N_{1}} y_{n} \cdot\left(\xi_{1}\right)^{n}-m \cdot\left(R_{0}+0,5 \cdot h\right)^{-1} \cdot \sum_{n=0}^{N_{1}} x_{n} \cdot\left(\xi_{1}\right)^{n}=0 \\
& R_{0}^{-1} \cdot \sum_{n=0}^{N_{1}} y_{n} \cdot n \cdot\left(-\xi_{1}\right)^{n-1}-\left(R_{0}-0,5 \cdot h\right)^{-1} \cdot \sum_{n=0}^{N_{1}} y_{n} \cdot\left(-\xi_{1}\right)^{n}-m \cdot\left(R_{0}-0,5 \cdot h\right)^{-1} \cdot \sum_{n=0}^{N_{1}} x_{n} \cdot\left(-\xi_{1}\right)^{n}=0 \\
& i \cdot k \cdot \sum_{n=0}^{N_{1}} x_{n} \cdot\left(\xi_{1}\right)^{n}+R_{0}^{-1} \cdot \sum_{n=0}^{N_{1}} z_{n} \bullet n \cdot\left(\xi_{1}\right)^{n-1}=0 ; \\
& i \cdot k \cdot \sum_{n=0}^{N_{1}} x_{n} \cdot\left(-\xi_{1}\right)^{n}+R_{0}^{-1} \cdot \sum_{n=0}^{N} z_{n} \bullet n \cdot\left(-\xi_{1}\right)^{n-1}=0 .
\end{aligned}
$$

The rest cof equations can be received, by substitution of decompositions (11) in equations (5) - (7) and by equated of coefficients at the fellow parameter $\xi[2-4,6]$ :

$$
\begin{aligned}
& x_{n+2} \bullet(n+2) \bullet(n+1)+x_{n+1} \bullet(n+1) \bullet(2 n+1)+x_{n} \bullet\left[n^{2}-m^{2} \bullet a_{3}-a_{5}+R_{0}^{2} \bullet\left(a_{7}-k^{2} \bullet a_{4}\right)\right]+x_{n-1} \cdot 2 \cdot R_{0}^{2} \bullet \\
& \bullet\left(a_{7}-k^{2} \cdot a_{4}\right)+x_{n-2} \cdot R_{0}^{2} \cdot\left(a_{7}-k^{2} \cdot a_{4}\right)+y_{n+1} \bullet(n+1) \bullet m \bullet\left(a_{1}+a_{3}\right)+y_{n} \bullet m \bullet\left[n \bullet\left(a_{1}+a_{3}\right)-a_{3}-a_{5}\right]+z_{n+1} \bullet \\
& \bullet i \bullet k \cdot\left(a_{2}+a_{4}\right) \cdot R_{0} \cdot(n+1)+z_{n} \cdot R_{0} \bullet i \bullet k \cdot\left[2 \cdot n \bullet\left(a_{2}+a_{4}\right)+a_{2}-a_{6}\right]+z_{n+1} \cdot R_{0} \bullet i \bullet k \bullet\left[(n-1) \bullet\left(a_{2}+a_{4}\right)+a_{2}-\right. \\
& \left.-a_{6}\right]=0 \\
& -x_{n+1} \bullet m \bullet\left(1+a_{8}\right) \bullet(n+1)-x_{n} \bullet m \cdot\left[n \bullet\left(1+a_{8}\right)+R_{0} \bullet a_{9}+1\right]-x_{n-1} \bullet R_{0} \bullet m \bullet a_{9}+y_{n+2} \bullet(n+2) \bullet(n+1)+y_{n} \bullet \\
& \bullet\left[n^{2}-R_{0} \bullet m^{2} \bullet a_{9}-1+R_{0}^{2} \bullet\left(a_{12}-k^{2} \bullet a_{11}\right)\right]+y_{n-1} \bullet R_{0} \bullet\left(2 \cdot R_{0} \bullet a_{12}-m^{2} \bullet a_{9}-2 \cdot R_{0} \bullet a_{11} \bullet k^{2}\right)-z_{n} \bullet R_{0} \bullet i \bullet k \bullet \\
& \cdot m \cdot\left(a_{10}+a_{11}\right)-z_{n-1} \cdot R_{0} \bullet i \cdot k \cdot m \cdot\left(a_{10}+a_{11}\right)=0 \\
& x_{n+1} \bullet i \bullet k \bullet\left(1+a_{14}\right) \cdot R_{0} \bullet(n+1)+x_{n} \bullet i \bullet k \cdot R_{0} \bullet\left[2 \cdot n \bullet\left(1+a_{14}\right)+1+a_{15}\right]+x_{n-1} \bullet i \bullet k \cdot R_{0} \bullet\left[(n-1) \bullet\left(1+a_{14}\right)+\right. \\
& \left.+1+a_{15}\right]+y_{n} \bullet i \bullet k \bullet m \cdot R_{0} \bullet\left(a_{15}+a_{13}\right)+y_{n-1} \bullet i \bullet k \bullet m \bullet R_{0} \bullet\left(a_{15}+a_{13}\right)+z_{n+2} \bullet(n+1) \bullet(n+2)+z_{n+1} \bullet(n+1) \bullet \\
& \bullet(2 \cdot n+1)+z_{n} \cdot\left[n^{2}-m^{2} \cdot a_{13}+R_{0}^{2} \cdot\left(a_{17}-k^{2} \cdot a_{16}\right)\right]+z_{n-1} \cdot 2 \cdot R_{0}^{2} \cdot\left(a_{17}-k^{2} \cdot a_{16}\right)+z_{n-2} \cdot R_{0}^{2} \cdot\left(a_{17}-k^{2} \bullet\right. \\
& \left.\cdot a_{16}\right)=0 \text {, }
\end{aligned}
$$


where

It is necessary to use $3 \cdot\left(N_{1}+!\right)-6$ of equations (18) (20), but for $n=0$ and $n=1$ coefficients with negative indexes are equal to zero. Then in common with equations (12) - (17) the homogeneous system of $3 \cdot\left(N_{1}+1\right)$ equations relative to coefficients $x_{n}, y_{n}, z_{n}$ is formed. Then we expand the determinant of this system and let this determinant is equal zero we receive the characteristic equation for wave numbers $k$ of elastic waves with the mode $m$ in the orthotropic cylindrical shell.

\section{Conclusions}

In the paper were found the characteristic equation for wave numbers of elastic waves in thin orthotropic cylindrical shell with the help of the dynamic theory of the elasticity for the orthotrpic medium and of the hypothesis of thin shells.

\section{Acknowledgments}

The work was supported as part of research under State Contract no P242 of April 21, 2010, within the Federal Target Program "Scientific and scientific pedagogical personnel of innovative Russia for the 2009 - 2013".

\section{REFERENCES}

[1] S. G. Lekhnitsky. The theory of elasticity of anisotropic body. M.: Science. 1977. P. 416.

[2] A. A. Kleshchev. Against phase velocities of elastic waves in thin orthotropic cylindrical shell. // Coll. Proc. Russ. Acoust. Soc. XI session. M.: GEOS. 2001. V. 1. P. $241-244$.

[3] A. A. Kleshchev. Diffraction and propagation of waves in elastic mediums and bodies. S.-Pb.: Vlas. 2002. P. 156.

[4] A. A. Kleshchev. Diffraction, radiation and propagation of elastic waves. S.-Pb.: Profprint. 2006. P. 160.

[5] E. L. Shenderov. Radiation and scattering of sound. L.: Shipbuilding. 1989. P. 302.

[6] A. A. Kleshchev. Against phase velocities of elastic waves in thin transversely isotropic cylindrical shell. // Coll. Proc. Russ. Acoust. Soc. X session. M.: GEOS. 2000. V. 1. P. $206-210$ 